\title{
Isokinetic relationships
}

\author{
preface
}

\author{
Wolfgang Linert $\cdot$ Arthur Yelon
}

Received: 14 September 2012/ Accepted: 16 September 2012/Published online: 18 October 2012

(C) Springer-Verlag Wien 2012

Almost 90 years have passed since Constable reported a relationship between the activation energies and activation entropies measured in his investigation of the dehydrogenation of ethanol, using a series of different catalysts. Decreasing activation energies were found to be linearly related to increasing activation entropies and vice versa. Thus, the increase of reaction rate with a "better" catalyst, exhibiting lower activation energy, was not as large as anticipated, since it was partly compensated by the "disadvantageous" decrease in entropy changes. This led to the term "compensation effect". This effect, and similar ones relating to kinetics in other fields of science, were (partly independently) observed over subsequent decades and given various names such as $\theta$-rule, Meyer-Neldel rule and Zawadzki-Bretszanjder rule. They are frequently called "isokinetic relationships" (IKR), since the linear relation implies that the rates of all of Constable's catalytic reactions, for example, will be the same at an isokinetic (or Meyer-Neldel) temperature.

A major obstacle to the acceptance of the reality and the understanding of such effects has been that incorrect statistical analysis can easily produce effects that look similar, often with the isokinetic temperature within the experimental range, but that are simply artefacts due to experimental

W. Linert $(\bowtie)$

Institute of Applied Synthetic Chemistry, Vienna University

of Technology, Getreidemarkt 9, 1060 Vienna, Austria

e-mail: wlinert@mail.zserv.tuwien.ac.at;

wolfgang.linert@tuwien.ac.at

\section{A. Yelon}

Department of Engineering Physics and Regroupement

Québecois sur les Matériaux de Pointe (RQMP),

École Polytechnique Montréal, PO Box 6079 Station "CV",

Montreal, QC H3C 3A7, Canada errors; For example, it is a mistake to perform a linear regression analysis on temperature-dependent kinetic or equilibrium data for a given series of reactions, in order to obtain enthalpies and entropies, and then use these in a further linear regression analysis to prove the existence of an IKR or isoequilibrium relationship (IER). ${ }^{1}$ For a long time, another obstacle to the understanding of these effects was the failure to realize that they are observed whenever the activation energy in a system or in related systems is large compared with thermal and excitation energies. In contrast, the effect is not observed when the activation energies are small. In such cases, the entropy may be independent of activation energy or it may increase or decrease slightly.

To avoid confusion with statistical artefacts, and to present correctly proven real effects, the term IKR (including IER, if thermodynamic data rather than kinetic results are investigated) has been used in the literature. We have used this term as the description of the present issue. Based on experimental and theoretical results, we strongly believe, not only that there can be little doubt that these effects are real, but also that they can be advantageously used for understanding, predicting and analysing the temperature dependence of series of similar reactions. Constable may have been frustrated in his efforts to increase the rates of catalytic reactions, but it is reasonable to suppose that the existence of catalysis depends upon compensation.

The investigation of compensation is almost surely more prominent in chemistry than in any other field, and a large fraction of the papers in this issue are devoted to chemical subjects. However, as pointed out above, it is a general

\footnotetext{
${ }^{1}$ Support concerning rigorous analysis may be obtained from W. Linert.
} 
rule, which can be found in many other fields. The issue contains papers on diffusion in solids, electronic properties of semiconductors, especially amorphous semiconductors, but also of deep traps in crystalline semiconductors, relaxations in polymers and processes in biological macromolecules. By inviting and assembling papers by experts in various aspects of compensation, we hope to promote wider knowledge and understanding of these phenomena, and that this will lead to further research in this highly interesting area. 